\title{
Artículo
}

\section{Adopción de buenas prácticas en la educación virtual en la educación superior}

\author{
Rodrigo Durán ${ }^{\mathrm{a}, *}$, Christian Estay-Niculcar ${ }^{\mathrm{b}}$ y Humberto Álvarez ${ }^{\mathrm{c}}$ \\ a CSDC Systems, Estados Unidos de América \\ b Secretaría Nacional de Administración Pública (SNAP), Ecuador \\ ' Facultad de Ingeniería Industrial, Universidad Tecnológica de Panamá, Panamá, Panamá
}

\section{INFORMACIÓN DEL ARTÍCULO}

\section{Historia del artículo:}

Recibido el 27 de agosto de 2014

Aceptado el 9 de enero de 2015

On-line el 11 de marzo de 2015

\section{Palabras clave:}

Educación virtual

Buenas prácticas educativas

Formación universitaria

Competencias

\begin{abstract}
R E S U M E N
Las tendencias actuales apuntan a que las personas pretenden aprender y estudiar en entornos flexibles. Por ende, la educación virtual en la educación superior juega un rol fundamental para satisfacer esta tendencia. Los propósitos de esta investigación son: primero, explorar de forma descriptiva el potencial de la educación virtual; segundo, emplear el modelo de buenas prácticas educativas de Chickering y Gamson (1987) en la redacción de actividades virtuales; y tercero, determinar el impacto de estas prácticas en la didáctica y el aprendizaje. La investigación se realizó con el docente y 10 estudiantes del curso denominado Modelado y Simulación de Sistemas Dinámicos de la Maestría en Ciencias de Tecnología de la Información y Comunicación (TIC) en la Universidad Tecnológica de Panamá. La investigación realizada es descriptiva y los resultados reflejan que tanto el docente como sus estudiantes evaluaron favorablemente las actividades de educación virtual, redactadas a partir del uso de buenas prácticas educativas. Por ende, este tipo de estrategias dentro de la planificación y el diseño curricular en la educación virtual aportan resultados positivos. Sin embargo, se reconoce que la muestra del estudio es limitada $(n=10)$, por lo que es necesario ampliar la muestra en otras investigaciones y recoger más datos.
\end{abstract}

(c) 2014 Instituto de Ciencias de la Educación de la Universidad de Oviedo. Publicado por Elsevier España, S.L.U. Este es un artículo Open Access bajo la licencia CC BY-NC-ND (http://creativecommons.org/licenses/by-nc-nd/4.0/).

\section{Adoption of good virtual education practices in higher education}

\section{A B S T R A C T}

Current trends suggest that people seek to learn and to study in flexible environments; therefore, virtual education in higher education plays a fundamental role to meet this trend. The aims of this study are: first, to descriptively examine the potential of virtual education, second, to use Chickering and Gamson (1987) good educational practices model to compose virtual activities, and third to determine the impact of these practices in teaching and learning. The study was conducted with the teacher and 10 students enrolled in a class named Model and Simulation of Dynamic Systems from the Master of Sciences in Information and Communication Technology at Universidad Tecnologica de Panama. This is a descriptive study, and the results show that both the teacher and his students favorably evaluated activities that consisted of using good educational practices, through virtual education. Therefore, this type of strategy for virtual education planning and curriculum design provides positive results. However, as the authors acknowledge that the sample is limited $(\mathrm{n}=10)$, further studies with larger samples are required in order to collect more data.

(C) 2014 Instituto de Ciencias de la Educación de la Universidad de Oviedo. Published by Elsevier España, S.L.U. This is an open access article under the CC BY-NC-ND license (http://creativecommons.org/licenses/by-nc-nd/4.0/).

\footnotetext{
* Autor para correspondencia. 555 Winderley Place Suite 300 Maitland, Florida 32751 Estados Unidos de América. Correo electrónico: ralduran@hotmail.com (R. Durán).
} 


\section{Introducción}

Las tecnologías de información y la comunicación (TIC) no solamente representan un instrumento o un nuevo medio de información y comunicación. Es importante tomar en consideración que generan un nuevo espacio social y, por ende, un nuevo espacio educativo conocido como educación virtual (Echeverría, 2002).

La educación virtual es un tipo de modalidad que representa una alternativa ideal para un importante grupo de estudiantes, ya que, por ejemplo, este tipo de educación les permite compatibilizar su actividad laboral y familiar con su formación, al desarrollarla en su propio domicilio. Algunas causas que originan la selección de la educación virtual como opción de aprendizaje son: el alejamiento de los centros de estudio superiores, la limitada disponibilidad de tiempo del estudiante y que el alumno presenta algún tipo de discapacidad que le impide acceder a las ofertas académicas presenciales (Silvio, 2006).

Cabe destacar que la educación virtual posee características que la diferencian en gran medida de la educación presencial. Por un lado, existe una mayor autonomía e independencia que disfruta el alumnado para el desarrollo de su proceso de aprendizaje, al ser el estudiante quien marca su ritmo de trabajo. Por el otro, muchos de los estudiantes conceden un carácter más práctico a sus objetivos de aprendizaje, debido a que este tipo de alumno desarrolla una actividad laboral vinculada a sus estudios formales, lo que favorece ampliamente su motivación intrínseca (Junta de Andalucía, 2012).

Suárez y Anaya (2004) señalan que estas mismas características también exigen del estudiante una mayor actividad autorregulada, responsabilidad y compromiso. Sin embargo, la educación virtual los limita a establecer relaciones y situaciones de aprendizaje compartido o cooperativo convencionales con otros participantes, que enriquecen la experiencia cognitiva y que son propias de la educación tradicional. No obstante, este último aspecto está siendo minimizado a partir de la correcta utilización de las TIC y, más concretamente, de las plataformas virtuales que cuentan con recursos tales como: foros, correo electrónico, hipervínculos a páginas web, enlaces a videoconferencias, entre otros.

La educación virtual en la educación superior ha sido tema de interés en muchas latitudes, incluyéndose como estrategia en iniciativas tales como: el Plan e-Europa aprobado por los primeros ministros de la Unión Europea en la cumbre de Lisboa celebrada los días 23 y 24 de mayo del 2000 (Europe-Summaries of EU Legislation, 2014), el proyecto sobre Educación Superior Virtual y a Distancia por parte del Instituto Internacional de la UNESCO para la educación superior en América Latina y el Caribe en el año 2002 (ANUIESUNESCO, 2004) y la Ley 30 del 20 de julio del 2006 que crea el Sistema Nacional de Evaluación y Acreditación para el Mejoramiento de la Calidad de la Educación Universitaria (CONEAUPA) en la República de Panamá, en la que se establece la importancia de la educación virtual como modalidad de enseñanza-aprendizaje universitaria (CONEAUPA, 2014).

La situación de la educación virtual en la educación superior de Panamá queda reflejada en el último informe emitido por la Comisión Técnica de Fiscalización de Panamá (CTF), entidad que, a febrero del 2014, resume que de un total de 745 carreras aprobadas pertenecientes a 32 universidades particulares, un subtotal de 9 carreras se ofrecen exclusivamente bajo la virtualidad (1\% del total), un subtotal de 42 carreras han sido aprobadas bajo diferentes modalidades incluyendo la virtualidad ( $6 \%$ del total) y un subtotal de 703 carreras han sido aprobadas bajo diferentes modalidades excluyendo la virtualidad ( $94 \%$ del total) (CTF, 2014).

\section{La importancia de la educación virtual en la educación superior}

La educación virtual es considerada como una modalidad que asume la educación tradicional producto de la globalización, de tal forma que la educación virtual es vista como el resultado de las TIC y la creación de sistemas de acceso a la red (Rama, 2006).

Las TIC han promovido la virtualización de la educación superior como un modelo de enseñanza y aprendizaje que incorpora la tecnología y la adquisición de competencias por parte de los alumnos. Además, permite organizar los aprendizajes necesarios y clasificar y definir las diferentes demandas de los profesionales en el mercado laboral (Silvio, 2000; Luque, 2004; Pastor y Santaolalla, 2005; Tunnermann, 2008; Rama, 2014).

Las primeras experiencias de esta modalidad se registraron en Inglaterra con la Open University que se constituyó como una de las instituciones pioneras en el uso de las tecnologías en la educación (García, 1999). La experiencia educativa de la Open University fue replicada en Europa, así como en otras universidades tales como la Universidad Nacional de Educación a Distancia (UNED), en España y FernUniversität, en Alemania, las cuales comenzaron a incursionar en la educación virtual durante la década de los setenta del siglo xx. Posteriormente, se crean instituciones homólogas en Irlanda, Suecia, Bélgica, Francia, Portugal y Noruega en la década de los ochenta del siglo xx. En América Latina, iniciativas pioneras de educación virtual en la educación superior fueron desarrolladas, por citar casos específicos, en el Instituto Tecnológico y de Estudios Superiores de Monterrey, en los Estados Unidos Mexicanos (año 1989), Universidad Abierta y a Distancia de Panamá, en la República de Panamá (año 1994) y la Universidad Federal de Santa Catarina, en la República del Brasil (año 1995)(Fernández y Vallejo, 2014). De igual forma, se han creado asociaciones o conglomerados en varias regiones o países del mundo que fomentan la educación virtual en la educación superior, destacándose: European Association of Distance Teaching Universities (EADTU), African Distance Learning Association (ADLA), Distance Education Association of New Zealand (DEANZ), National Association of Distance Education Organizations of South Africa (NADEOSA), United States Distance Learning Association (USDLA), Le Réseau d'enseignement francophone à distance du Canada (REFAD), Associação Brasileira de Educação a Distância (ABED) y la Asociación Colombiana de educación superior a Distancia (ACESAD) (Asociaciones de Educación a Distancia, 2014), entre otras.

En Iberoamérica, la educación virtual en la educación superior está desempeñando un papel relevante, ya que el Horizon Project resume las siguientes tendencias para el periodo 2012-2017 (Durall, Gros, Maina, Johnson, Adams, 2012): primero, las personas pretenden poder trabajar, aprender y estudiar cuando quieran y desde donde quieran. Segundo, la multitud de recursos y relaciones disponibles en Internet obligan a revisar el papel de los profesores en los procesos de creación de sentido, asesoramiento y acreditación. Tercero, las tecnologías que se utilizan están cada vez más basadas en la computación en nube y las nociones de apoyo a las TIC están descentralizadas. Cuarto, los cambios en la enseñanza universitaria inducen a la mayoría de las universidades a situar la capacitación de los docentes como un elemento estratégico en la calidad de la docencia. Finalmente, el ámbito laboral es cada vez más colaborativo, lo que conlleva cambios en el modo de estructurar los proyectos estudiantiles.

\section{Las buenas prácticas como fin}

El término o concepto de buenas prácticas fue acuñado por Hammer (1990) y se define como una manera de realizar un trabajo que produce un buen resultado. Una práctica exitosa es reconocida por ser innovadora, replicable, evaluada y porque transforma desde el ejercicio responsable de su autonomía. Adicionalmente, presupone un acto explícito de decisión que compromete recursos institucionales (The Global University Network for Innovation, 2014).

La buena práctica en la universidad se define como una experiencia (programa, proyecto) que favorece significativamente la 
pertinencia social de las instituciones de educación superior, promoviendo un papel activo en la construcción de una sociedad más justa y sostenible social, política, cultural, medioambiental y económicamente (Red Telescopi, 2014).

El concepto de buenas prácticas ha estado presente en el núcleo fundamental de la investigación educativa desde la segunda mitad del siglo xx (Zabalza, 2012). Incluso, Van Haecht (1998) señala que la idea de buscar referentes de buenas prácticas en instituciones del extranjero, consideradas modelos de rol, figuraba ya en manuscritos de inicios del siglo XIX, específicamente en los provenientes de L'Esquisse d'un ouvrage sur l'education comparée, de Jullien del año 1816.

El grupo de Investigación y Multimedia de la Universidad Autónoma de Barcelona (2014) define las buenas prácticas docentes como las intervenciones educativas que facilitan el desarrollo de actividades de aprendizaje en las que se logren con eficiencia los objetivos formativos previstos y también otros aprendizajes de alto valor educativo, señalando para ello, algunos de los siguientes indicadores: significación para los estudiantes, implicación del alumnado, tratamiento de la diversidad tanto en los contenidos que se presentan como en las estrategias de actuación que implican, nivel de operaciones cognitivas implicadas, participación social y trabajo colaborativo.

Otras definiciones sobre buenas prácticas docentes son las propuestas por de Pablos y Jiménez (2007) y Cabero y Romero (2010). Por un lado, de Pablos y Jiménez (2007, pag.26) definen las buenas prácticas docentes como un proceso de cambio que debe incidir en las formas de construcción del conocimiento, en la configuración de nuevos entornos de enseñanza y aprendizaje y en la transformación de la cultura de la Academia y del docente. Por otro lado, Cabero y Romero (2010, pag.285) definen las buenas prácticas docentes como las intervenciones educativas de los profesores y las instituciones que facilitan el desarrollo de actividades que permiten que los estudiantes alcancen objetivos, capacidades y competencias establecidas.

Instituciones internacionales como la Organización de las Naciones Unidas para la Educación, la Ciencia y la Cultura (UNESCO), el Bureau International d'Education (BID), la Organisation pour la Sécurité et la Coopération en Europe (OSCE), el Bureau des Institutions Démocratiques et des Droits de l'Homme (BIDDH) y el Consejo de Europa han insistido en la importancia de compilar buenas prácticas en el ámbito educativo que sirvan como base epistémica para el desarrollo de las políticas educativas (Zabalza, 2012).

En América Latina, el Programa de Promoción de la Reforma Educativa en América Latina y el Caribe (PREAL, 2014) ha publicado una compilación de buenas prácticas existentes en los 18 diferentes países de la región, que pertenecen a la base de datos denominada Mejores Prácticas de Política Educacional y Reforma Educativa. Dicha publicación se considera parte de los esfuerzos por mejorar la educación y obtener mejores resultados en este renglón. Las buenas prácticas de esta iniciativa están agrupadas en los siguientes ejes temáticos: formación docente, gestión, materiales curriculares, mantenimiento y mejoramiento de la infraestructura educativa, incorporación de las nuevas tecnologías y sistemas de evaluación.

\section{Selección del conjunto de buenas prácticas educativas}

En la tabla 1 se resumen y comparan 3 modelos de buenas prácticas educativas: Chickering y Gamson (1987), Alexander (1997) y Coffield y Edward (2009) con el propósito de establecer un conjunto de buenas prácticas aplicables al currículum universitario.

Según los autores de la investigación, quienes cuentan con experiencia en la planificación y diseño de cursos virtuales, trabajar una lista puntual de 7 buenas prácticas como la propuesta por Chickering y Gamson (1987) es más sencillo de aplicar en las actividades de aprendizaje que emplear un conjunto de interrogantes o un conjunto de dimensiones como las propuestas por Alexander (1997) y Coffield y Edward (2009), respectivamente.

Para darle valor científico al juicio de los autores, se investigó y confirmó que el modelo de Chickering y Gamson (1987) ha sido utilizado en importantes estudios sobre educación a distancia o virtual, destacándose los trabajos realizados por Graham, Cagiltay, Lin, Craner y Duffy (2001), Hutchins (2003), Bangert (2004), Tobin (2004), Dixon (2012), Babb, Stewart y Johnson (2013) y Cakiroglu (2014). En estos estudios se aplican los principios de Chickering y Gamson (1987), concebidos originalmente para la educación tradicional, en contextos o entornos virtuales.

Por ende, la propuesta de Chickering y Gamson (1987) será la seleccionada para el desarrollo de este estudio.

Tabla 1

Modelo de buenas prácticas educativas

\begin{tabular}{|c|c|c|c|}
\hline Criterios Autores & Chickering y Gamson (1987) & Alexander (1997) & Coffield y Edward (2009) \\
\hline Antecedentes & No aplica & No aplica & Profundiza el trabajo de Alexander \\
\hline Fundamento del modelo & $\begin{array}{l}\text { Siete principios de buenas prácticas } \\
\text { educativas }\end{array}$ & $\begin{array}{l}\text { Utiliza propuestas pedagógicas de } \\
\text { manera reflexiva, elaboradas por los } \\
\text { profesores que interaccionan con ellas }\end{array}$ & $\begin{array}{l}\text { Se basa en el contexto, en el } \\
\text { conocimiento, el currículum, la } \\
\text { pedagogía, la evaluación, la gestión y la } \\
\text { sociedad }\end{array}$ \\
\hline ¿Qué describe al modelo? & $\begin{array}{l}\text { Listado puntual de } 7 \text { principios de } \\
\text { buenas prácticas educativas }\end{array}$ & Listado de interrogantes & Listado de dimensiones \\
\hline Detalle del modelo & $\begin{array}{l}\text { Práctica 1: Promover las relaciones } \\
\text { entre profesores y alumnos } \\
\text { Práctica 2: Desarrollar dinámicas de } \\
\text { cooperación entre los alumnos } \\
\text { Práctica 3: Aplicar técnicas activas para } \\
\text { el aprendizaje } \\
\text { Práctica 4: Permitir procesos de } \\
\text { retroalimentación } \\
\text { Práctica 5: Enfatizar el tiempo de } \\
\text { dedicación a la tarea } \\
\text { Práctica 6: Comunicar altas } \\
\text { expectativas } \\
\text { Práctica 7: Respetar la diversidad de } \\
\text { formas de aprender }\end{array}$ & $\begin{array}{l}\text { ¿Quiénes, la administración u otros } \\
\text { grupos de poder, proponen una buena } \\
\text { práctica, para qué y con qué } \\
\text { consecuencias? } \\
\text { ¿Cómo una buena práctica concuerda o } \\
\text { cuestiona la propia y los valores que la } \\
\text { sostienen? } \\
\text { ¿Qué soportes y evidencias basadas en la } \\
\text { investigación educativa relevante la } \\
\text { avalan? } \\
\text { ¿Qué grado de utilidad puede tener una } \\
\text { práctica presentada como buena para el } \\
\text { profesorado y su contexto de trabajo? } \\
\text { ¿Qué concepciones de una buena } \\
\text { enseñanza y aprendizaje son las que } \\
\text { están inspirando la práctica de un } \\
\text { docente? }\end{array}$ & $\begin{array}{l}\text { Peculiaridades organizativas } \\
\text { Alumnado } \\
\text { Historia previa, programa formativo } \\
\text { Tipo de presupuestos, valores } \\
\text { Conocimientos y capacidades } \\
\text { organizativas y profesionales de la } \\
\text { práctica } \\
\text { Selección de contenidos y aprendizajes } \\
\text { Planificación, secuencia y evaluación de } \\
\text { la práctica } \\
\text { Habilidades, creencias y valores de los } \\
\text { profesores } \\
\text { Influencias, relaciones y adecuación de } \\
\text { una buena práctica con el mercado } \\
\text { laboral }\end{array}$ \\
\hline
\end{tabular}


Tabla 2

Matriz de autoevaluación del docente

Por cada celda, responder a la siguiente interrogante:

¿La narrativa de la actividad de educación virtual $n$, llena las expectativas de la práctica $m$ de Chickering y Gamson (1987)?

\begin{tabular}{|c|c|c|c|c|c|c|c|c|c|}
\hline Número & Actividad & Práctica 1 & Práctica 2 & Práctica 3 & Práctica 4 & Práctica 5 & Práctica 6 & Práctica 7 & $\begin{array}{l}\text { Promedio de } \\
\text { actividad }\end{array}$ \\
\hline 1 & Actividad 1 & $\mathrm{E}_{11}$ & $\mathrm{E}_{12}$ & $\mathrm{E}_{13}$ & $\mathrm{E}_{14}$ & $\mathrm{E}_{15}$ & $\mathrm{E}_{16}$ & $\mathrm{E}_{17}$ & $\mathrm{P}_{1 m}$ \\
\hline 2 & Actividad 2 & $\mathrm{E}_{21}$ & $\mathrm{E}_{22}$ & $\mathrm{E}_{23}$ & $\mathrm{E}_{24}$ & $\mathrm{E}_{25}$ & $\mathrm{E}_{26}$ & $\mathrm{E}_{27}$ & $\mathrm{P}_{2 m}$ \\
\hline 3 & Actividad 3 & $\mathrm{E}_{31}$ & $\mathrm{E}_{32}$ & $\mathrm{E}_{33}$ & $\mathrm{E}_{34}$ & $\mathrm{E}_{35}$ & $\mathrm{E}_{36}$ & $\mathrm{E}_{37}$ & $\mathrm{P}_{3 m}$ \\
\hline 4 & Actividad 4 & $\mathrm{E}_{41}$ & $\mathrm{E}_{42}$ & $\mathrm{E}_{43}$ & $\mathrm{E}_{44}$ & $\mathrm{E}_{45}$ & $\mathrm{E}_{46}$ & $\mathrm{E}_{47}$ & $\mathrm{P}_{4 m}$ \\
\hline$n$ & Actividad n & $\mathrm{E}_{n 1}$ & $\mathrm{E}_{n 2}$ & $\mathrm{E}_{n 3}$ & $\mathrm{E}_{n 4}$ & $\mathrm{E}_{n 5}$ & $\mathrm{E}_{n 6}$ & $\mathrm{E}_{n 7}$ & $\mathrm{P}_{n m}$ \\
\hline Promedio de buena práctica & $\mathrm{P}_{n 1}$ & $\mathrm{P}_{n 2}$ & $\mathrm{P}_{n 3}$ & $\mathrm{P}_{n 4}$ & $\mathrm{P}_{n 5}$ & $\mathrm{P}_{n 6}$ & $\mathrm{P}_{n m}$ & $P_{n+1, m+1}$ & \\
\hline
\end{tabular}

Los 3 propósitos de la investigación se describen a continuación: primero, explorar de forma descriptiva el potencial de la educación virtual en la educación superior, como alternativa de calidad en el proceso de enseñanza y aprendizaje; segundo, emplear el modelo de buenas prácticas educativas de Chickering y Gamson (1987) para redactar y evaluar las actividades que se desarrollan en modalidad virtual, específicamente en un curso de maestría; y tercero, determinar el impacto de estas prácticas en la didáctica del docente y el aprendizaje del alumno.

\section{Método}

\section{Participantes}

La unidad de análisis es el docente y el estudiante. A partir de sus aportes, se probará la efectividad de redactar las actividades de educación virtual a partir de las buenas prácticas educativas de Chickering y Gamson (1987). La metodología se desarrolla a partir de un estudio de caso en el que participaron el docente y sus 10 estudiantes inscritos en el curso de Modelado y Simulación de Sistemas Dinámicos de la Maestría en Ciencias de Tecnología de la Información y Comunicación ofrecida por la Universidad Tecnológica de Panamá.

En cuanto a los participantes del estudio de caso, este grupo de 10 estudiantes presentan las siguientes características: la media de los participantes es de 26,2 años, con una desviación estándar de 2,99 años. El género de los participantes está distribuido en partes iguales. El $80 \%$ de los participantes contaban solamente con estudios de pregrado antes de inscribirse al programa de maestría, mientras que solo el $20 \%$ contaba con estudios de posgrado, antes de inscribirse al programa.

El antecedente laboral de los participantes antes de inscribirse al programa de maestría se detalla a continuación: un estudiante contaba con 8 años de experiencia laboral, 3 estudiantes contaban con 4 años de experiencia laboral, 3 estudiantes contaban con un año de experiencia laboral, 2 estudiantes contaban con menos de un año de experiencia laboral y solo un estudiante no contaba con experiencia laboral previa. El 100\% de los participantes con experiencia laboral han desarrollado su carrera en el sector tecnológico.

\section{Instrumentos}

La presente investigación utiliza 3 instrumentos que se describen a continuación:

1. Matriz de autoevaluación del docente

Este instrumento representado en la tabla 2 pretende calcular el promedio del docente tras evaluar cómo las buenas prácticas educativas de Chickering y Gamson (1987) influyeron en la redacción de las actividades de educación virtual. De la celda $(n+1, m+1)$ que se encuentra en la (tabla 2) se puede leer el promedio de esta autoevaluación, el cual representa el indicador clave del instrumento.
La estructura del instrumento consta de una matriz de $(n+1)$ filas, donde $n$ es el conjunto de actividades de educación virtual redactadas a partir del modelo seleccionado y de $(m+1)$ columnas, donde $m$ representa las 7 prácticas educativas de Chickering y Gamson (1987). En la fila $(n+1)$ y la columna $(m+1)$ se registran en sus correspondientes celdas los resultados de calcular el promedio por actividad (filas) y por práctica (columnas).

Modo de cumplimentación: El número de celdas con respuesta a la pregunta de investigación se obtiene tras multiplicar el número de $n$ filas (actividades) por el número de $m$ columnas (buenas prácticas). Por ejemplo, si $n=5$ y $m=7$, donde $m$ siempre es constante, el total de respuestas o celdas equivale a 35 .

Por cada celda $(n, m)$ de la tabla 2 se debe elegir un solo valor del rango [ 1 a 5]. Si la actividad dependiendo de su redacción no llenó las expectativas de la práctica, se selecciona el valor numérico 1 . Si la actividad cumplió escasamente con la práctica, se selecciona el valor numérico 2 . Si la actividad cumple parcialmente con la práctica, se selecciona el valor numérico 3. Si la actividad cumple con la práctica, se selecciona el valor numérico 4 . Finalmente, si la actividad cumple en gran medida con la práctica, se selecciona el valor numérico 5.

Se procede a explicar el significado de las variables de la tabla 2. En primer lugar, $\mathrm{E}_{11} \ldots \mathrm{E}_{n m}$ representan celdas con valores numéricos enteros en el conjunto $=\{1,2,3,4,5\}$, cada celda puede contener un solo valor de ese conjunto. En segundo lugar, el promedio por actividad se determina por la expresión:

$P_{1 m}=\frac{\left(E_{11}+E_{12}+E_{13}+E_{14}+E_{15}+E_{16}+E_{17}\right)}{m}$

Para $\mathrm{P}_{2 m}, \mathrm{P}_{3 m}, \mathrm{P}_{4 m}, \mathrm{P}_{n m}$ se aplica la misma fórmula, solamente se varía la fila representada en el par $(n, m)$, donde $n$ es la fila y $m$ es la columna. En tercer lugar, el promedio por buena práctica se determina por la expresión:

$P_{n 1}=\frac{\left(E_{11}+E_{21}+E_{31}+E_{41}+E_{51}\right)}{n}$

Para $\mathrm{P}_{n 2}, \mathrm{P}_{n 3}, \mathrm{P}_{n 4}, \mathrm{P}_{n 5}, \mathrm{P}_{n 6}, \mathrm{P}_{n m}$ se aplica la misma fórmula de $\mathrm{P}_{n 1}$, solamente se varía la columna representada en el par $(n, m)$, donde $n$ es la fila y $m$ es la columna. Finalmente, el resultado de la proyección de evaluar las actividades con relación a las buenas prácticas o $\mathrm{P}_{n+1, m+1}$ se puede calcular de 2 formas:

$P_{n+1, m+1}=\frac{\left(P_{1 m}+P_{2 m}+P_{3 m}+P_{4 m}+\cdots+P_{n m}\right)}{n}$

$P_{n+1, m+1}=\frac{\left(P_{n 1}+P_{n 2}+P_{n 3}+P_{n 4}+\cdots+P_{n m}\right)}{m}$

2. Instrumento para la evaluación por parte de los alumnos

Este instrumento representado en la figura 1 pretende que el estudiante evalúe sus aprendizajes a partir de las actividades de educación virtual. 


\section{EVALUACIÓN DE LAS ACTIVIDADES DE APRENDIZAJE}

Objetivo del instrumento: Determinar si las diferentes actividades utilizadas en la fase virtual del curso Modelo de Simulación de Sistemas Dinámicos, contribuye al aprendizaje del estudiante.

Sección 1 - Preguntas generales

1) Edad:

2) Años de experiencia laboral:

3) Máximo nivel de escolaridad alcanzado a la fecha:
a. Licenciatura o Ingeniería
b. Postgrado
c. Maestría
d. Doctorado
e. Post-Doctorado

4) Seleccione la especialidad en la cual usted ha desarrollado su Carrera según la clasificación UNESCO
a. Lógica
b. Matemática
c. Astronomía y Astrofísica
d. Física
e. Química
f. Ciencias de la Vida
$\ldots$
g. Tecnología

Sección 2 - Favor responder a la pregunta de investigación. En la columna de evaluación, el estudiante califica con uno (1) si la actividad no aporta en sus aprendizajes; con dos (2), si la actividad contribuye escasamente en sus aprendizajes; con tres (3) si la actividad aporta parcialmente en sus aprendizajes; con un (4) si la actividad aporta en sus aprendizajes; y con (5) si la actividad contribuye en gran medida en sus aprendizajes.

1) ¿Pudo usted comprender la narrativa de la actividad de educación virtual n?

\begin{tabular}{|l|l|c|}
\hline ID & Actividad & Evaluación \\
\hline 1 & Descripción de la actividad 1 & $\mathrm{E}_{11}$ \\
\hline 2 & Descripción de la actividad 2 & $\mathrm{E}_{12}$ \\
\hline 3 & Descripción de la actividad 3 & $\mathrm{E}_{13}$ \\
\hline 4 & Descripción de la actividad 4 & $\mathrm{E}_{14}$ \\
\hline \multicolumn{2}{|l|}{$\ldots$} & $\mathrm{E}_{1 n}$ \\
\hline$n$ & Descripción de la actividad $n$ & \\
\hline
\end{tabular}

Favor remitir el documento por vía electrónica al correo del docente titular del curso

Figura 1. Instrumento para la evaluación de las actividades por los estudiantes

El instrumento consta de 2 secciones. En la primera sección se formulan 4 preguntas relacionadas con el perfil del estudiante y en la sección 2 se incluye una pregunta de investigación que se repite dependiendo del número de actividades de educación virtual.

La sección 1 se completa respondiendo a las 4 preguntas que se le formulan al estudiante. La sección 2 se completa de la siguiente forma: por cada actividad de educación virtual, el estudiante debe responder a la pregunta de investigación, eligiendo un solo valor del rango [1-5]. Continuando con la sección 2, si la actividad de educación virtual $n$ no aporta al aprendizaje del estudiante, se selecciona el valor numérico 1 . Si esta actividad contribuye escasamente al aprendizaje del alumno, se selecciona el valor numérico 2 . Si la actividad contribuye medianamente al aprendizaje del estudiante, se selecciona el valor numérico 3. Si la actividad aporta al aprendizaje del estudiante, se selecciona el valor numérico 4. Finalmente, si la actividad contribuye en gran medida al aprendizaje del alumno, se selecciona el valor numérico 5 .

Se procede a explicar el significado de las variables de la figura 1. $\mathrm{E}_{11}, \mathrm{E}_{12}, \mathrm{E}_{13}, \mathrm{E}_{14}, \ldots \mathrm{y} \mathrm{E}_{1 n}$ representan celdas con valores números enteros en el conjunto $=\{1,2,3,4,5\}$, donde cada celda puede contener un solo valor del citado conjunto para responder a la pregunta de la sección 2 del instrumento.
3. Matriz con el resumen de las evaluaciones de los alumnos

Este instrumento representado en la tabla 3 pretende calcular el promedio por caso o estudiante a partir de su evaluación de las actividades de educación virtual, el promedio obtenido por cada actividad de educación virtual y el promedio de promedios de los estudiantes participantes en el estudio de caso. El indicador clave de este instrumento se obtiene de la celda $(n+1, m+1)$ que se encuentra en la tabla 3.

La estructura del instrumento consta de una matriz de $(n+1)$ filas y de $(m+1)$ columnas. Con respecto a las filas, $n$ es el número de estudiantes participantes en el estudio de caso y la fila $(n+1)$ representa el promedio por actividad. Con respecto a las columnas, $m$ es el número de actividades que han sido evaluadas por los estudiantes y la columna $(m+1)$ representa el promedio por caso o estudiante.

Se procede a explicar el significado de las variables de la tabla 3. En primer lugar, $\mathrm{E}_{11} \ldots \mathrm{E}_{n m}$ representan celdas con valores numéricos enteros en el conjunto $=\{1,2,3,4,5\}$, cada celda puede contener un solo valor de ese conjunto. En segundo lugar, el promedio por caso de la tabla 3 se calcula de la misma forma que el promedio por actividad de la figura $1 \mathrm{y}$ el promedio por actividad de la tabla 3 se calcula de la misma forma que el promedio de la 
Tabla 3

Matriz de consolidación de las diferentes evaluaciones de los estudiantes

\begin{tabular}{|c|c|c|c|c|c|c|}
\hline \multirow[t]{2}{*}{ Casos } & \multicolumn{5}{|c|}{ Actividades de educación virtual } & \multirow[t]{2}{*}{ Promedios por caso } \\
\hline & 1 & 2 & 3 & 4 & $m$ & \\
\hline Estudiante 1 & $\mathrm{E}_{11}$ & $\mathrm{E}_{12}$ & $\mathrm{E}_{13}$ & $\mathrm{E}_{14}$ & $\mathrm{E}_{1 m}$ & Promedio $\mathrm{E}_{1}$ \\
\hline Estudiante 2 & $\mathrm{E}_{21}$ & $\mathrm{E}_{22}$ & $\mathrm{E}_{23}$ & $\mathrm{E}_{24}$ & $\mathrm{E}_{2 m}$ & Promedio $\mathrm{E}_{2}$ \\
\hline Estudiante 3 & $E_{31}$ & $\mathrm{E}_{32}$ & $\mathrm{E}_{33}$ & $\mathrm{E}_{34}$ & $\mathrm{E}_{3 m}$ & Promedio $\mathrm{E}_{3}$ \\
\hline Estudiante 4 & $\mathrm{E}_{41}$ & $\mathrm{E}_{42}$ & $\mathrm{E}_{43}$ & $\mathrm{E}_{44}$ & $\mathrm{E}_{4 m}$ & Promedio $\mathrm{E}_{4}$ \\
\hline Estudiante 5 & $E_{51}$ & $\mathrm{E}_{52}$ & $\mathrm{E}_{53}$ & $\mathrm{E}_{54}$ & $\mathrm{E}_{5 m}$ & Promedio $\mathrm{E}_{5}$ \\
\hline Estudiante $\mathrm{n}$ & $\mathrm{E}_{n 1}$ & $\mathrm{E}_{n 2}$ & $\mathrm{E}_{n 3}$ & $\mathrm{E}_{n 4}$ & $\mathrm{E}_{n m}$ & Promedio $\mathrm{E}_{n}$ \\
\hline Promedio por actividad & $\begin{array}{l}\text { Promedio } \\
\text { actividad }_{1}\end{array}$ & $\begin{array}{l}\text { Promedio } \\
\text { actividad }_{2}\end{array}$ & $\begin{array}{l}\text { Promedio } \\
\text { actividad }_{3}\end{array}$ & $\begin{array}{l}\text { Promedio } \\
\text { actividad }_{4}\end{array}$ & $\begin{array}{l}\text { Promedio } \\
\text { actividad }_{m}\end{array}$ & $\begin{array}{l}\text { Promedio de Promedios } \\
(n+1, m+1)\end{array}$ \\
\hline
\end{tabular}

buena práctica de la figura 1 . Finalmente, Promedio de promedios $=\left(\right.$ Promedio estudiante $_{1}+$ Promedio estudiante $_{2}+$ Promedio estudiante $_{3}+\ldots+$ Promedio estudiante $\left._{n}\right) / n$

Promedio de promedios $=\left(\right.$ Promedio $_{\text {actividad }_{1}}+$ Promedio $\operatorname{actividad}_{2}+$ Promedio $_{\text {actividad }}+\ldots+$ Promedio $_{3}$ actividad $\left._{m}\right) / m$.

Donde $n$ equivale al número de estudiantes y $m$ equivale al número de actividades de educación virtual.

\section{Procedimiento}

La investigación es descriptiva y se centra en utilizar los 7 principios de buena práctica educativa de Chickering y Gamson (1987) en la planificación y diseño de un curso de maestría de la Universidad Tecnológica de Panamá con 19 sesiones presenciales distribuidas en 12 semanas de clase.

El procedimiento siguió las siguientes fases: la primera fase consiste en redactar las actividades de educación virtual tomando como referencia las 7 buenas prácticas educativas de Chickering y Gamson (1987) listadas en la tabla 1; la segunda fase equivale a utilizar el formato de la tabla 2, de manera que el docente evalúe las actividades de educación virtual con respecto a las buenas prácticas educativas de Chickering y Gamson (1987); y la tercera fase consiste en aplicar el formato de la figura 1 , de manera que los estudiantes evalúen las actividades de educación virtual y el impacto que dichas actividades lograron en sus aprendizajes.

Como parte de la metodología se solicita al docente del curso la cumplimentación de la matriz de autoevaluación, al principio del periodo académico y al estudiante la cumplimentación del instrumento de evaluación, al final del periodo académico. En ambos instrumentos, las actividades de educación virtual fueron valoradas por ambos grupos de interés desde la perspectiva didáctica y de aprendizaje, respectivamente.

A continuación se presentan las acciones puntuales tomadas por el docente participante en el estudio de caso. En la primera acción el docente investigador seleccionó uno de los cursos de maestría que dicta en la Universidad Tecnológica de Panamá para realizar la investigación. En la segunda acción se invitó voluntariamente a los estudiantes a participar en el estudio. En la tercera acción el estudio se organizó de tal manera que el docente investigador autoevaluara, al principio del periodo académico, la adopción de las buenas prácticas educativas en la redacción de las actividades de aprendizaje virtuales y que cada estudiante evaluara, al final del periodo académico, el impacto que las actividades de educación virtual habían logrado en sus aprendizajes. Finalmente, en la cuarta acción la metodología se aplicó a un mismo curso.

La duración del estudio de caso es de 69 días hábiles y entre sus principales actividades destacan: la redacción de las actividades de educación virtual, a partir de las buenas prácticas de Chickering y Gamson (1987), con una duración de 2 días hábiles, la evaluación de la redacción de las actividades de aprendizaje en función de las buenas prácticas educativas por el docente del curso, con una duración de 3 días hábiles, la implementación curricular incluyendo las actividades de educación virtual empleando las buenas prácticas educativas de Chickering y Gamson (1987), con una duración de 61 días hábiles y la evaluación de las actividades de educación virtual por los estudiantes participantes del estudio de caso, con una duración de 3 días hábiles.

La figura 2 enumera las técnicas, recursos y actividades empleadas en la fase virtual del curso Modelado y Simulación de Sistemas Dinámicos.

Para la redacción de las actividades de educación virtual empleando las 7 buenas prácticas de Chickering y Gamson (1987) se utilizó como descripción base las 4 actividades listadas en la figura 2. La figura 3 lista las 4 actividades que han sido reescritas tras la adopción de los principios del modelo de Chickering y Gamson (1987). Además, esta figura es una representación visual de las actividades de aprendizaje al momento de acceder a la plataforma virtual.

Las descripciones de las 4 actividades de aprendizaje de la figura 3 fueron incluidas en los instrumentos 1 y 2 (tabla 2 y fig. 1 , respectivamente) con el propósito de que las actividades virtuales fueran evaluadas por el docente investigador y los estudiantes participantes del estudio de caso.

\section{Resultados}

Las 7 buenas prácticas educativas de Chickering y Gamson (1987) se evidencian en las 4 actividades de aprendizaje virtuales de la figura 3, de la siguiente forma: la práctica 1 de Chickering y Gamson (1987) se manifiesta en aquellos párrafos donde el docente señala los canales de comunicación a través de los cuales el estudiante puede realizar consultas. La práctica 2 se evidencia en los párrafos donde el docente invita al estudiante a colaborar con sus compañeros. La práctica 3 se adopta en los párrafos donde el docente recomienda las técnicas para la realización de la actividad. La práctica 4 se evidencia en los párrafos donde el docente señala los tiempos estimados de respuesta a las preguntas formuladas por los estudiantes. La práctica 5 se identifica en los párrafos donde el docente menciona el tiempo de dedicación de cada actividad. La práctica 6 se hace presente en los párrafos donde el docente comunica sobre las altas expectativas, específicamente cuando invita al estudiante a ser creativo y a cuidar la calidad de sus entregables. Finalmente, la práctica 7 se manifiesta en aquellos párrafos donde el docente le da libertad al alumno para generar sus propios formatos de entrega. La tabla 4 presenta los resultados de la autoevaluación realizada por el docente, al inicio del periodo académico.

A partir de la lectura de la tabla 4 se desprenden los siguientes resultados: primero, en el ámbito de las actividades, las actividades 1 y 2 obtuvieron un promedio de 4,43 y las actividades 3 y 4 alcanzaron un promedio de 4,29. Lo anterior significa que las 4 actividades cumplen con las prácticas educativas en cuanto a redacción. Segundo, a nivel de las prácticas, el promedio de las prácticas 1, 3 y 5 es de 5,00, lo que significa que las 4 actividades de aprendizaje fueron influidas en gran medida por estas 3 prácticas. Tercero, la 


\begin{tabular}{|c|c|}
\hline Variable & Modalidad Virtual \\
\hline Técnicas & $\begin{array}{l}\text { - } \quad \text { Lista de discusión } \\
\text { - } \quad \text { Grupos de interés } \\
\text { - } \quad \text { Correo electrónico }\end{array}$ \\
\hline Recursos & $\begin{array}{l}\text { - Plataforma Aula net de la Universidad Tecnológica de Panamá } \\
\text { - Material instruccional digital } \\
\text { Plataforma virtual humberto-r-alvarez-a.webs.com con } 106 \text { enlaces a } \\
\text { sitios de interés para el estudiante entre los que destacan el Harvard } \\
\text { Business for Educators y el Carnegie for the Advancement of } \\
\text { Teaching }\end{array}$ \\
\hline $\begin{array}{l}\text { Actividades } \\
\text { sin la } \\
\text { adopción } \\
\text { de las }\end{array}$ & $\begin{array}{l}\text { - Actividad 1: Estudio comparativo de documentos y análisis de una } \\
\text { experiencia, problema u oportunidad en su vida profesional como } \\
\text { problema social complejo. }\end{array}$ \\
\hline $\begin{array}{l}\text { buenas } \\
\text { prácticas } \\
\text { educativas } \\
\text { de } \\
\text { Chickering }\end{array}$ & $\begin{array}{l}\text { - Actividad 2: Búsqueda de al menos tres aplicaciones de dinámica de } \\
\text { sistemas en la literatura y comparación entre ellas en base a } \\
\text { metodología de trabajo, resultados obtenidos y aplicabilidad en } \\
\text { general. }\end{array}$ \\
\hline $\begin{array}{l}\text { y Gamson } \\
(1987)\end{array}$ & $\begin{array}{l}\text { - Actividad 3: Buscar en la literatura al menos dos referencias que } \\
\text { mencionen aspectos relativos en su investigación aplicando } \\
\text { conceptos de sistemas complejos y/o dinámica de sistemas y sus } \\
\text { conclusiones. En caso de no encontrar, hacer un análisis que sustente } \\
\text { si la dinámica de sistemas es o no aplicable en estos casos }\end{array}$ \\
\hline & $\begin{array}{l}\text { - Actividad 4: Análisis de diagrama causal de las variables operativas } \\
\text { de su propuesta de investigación y los resultados esperados. }\end{array}$ \\
\hline
\end{tabular}

Figura 2. Técnicas, recursos y actividades empleadas en la fase virtual

Tabla 4

Resultados de la autoevaluación del docente

\begin{tabular}{|c|c|c|c|c|c|c|c|c|}
\hline Actividad & Práctica 1 & Práctica 2 & Práctica 3 & Práctica 4 & Práctica 5 & Práctica 6 & Práctica 7 & $\begin{array}{l}\text { Promedio tras } \\
\text { relacionar la } \\
\text { actividad con las } 7 \\
\text { prácticas }\end{array}$ \\
\hline $\begin{array}{l}\text { Actividad } 1 \text { - Ver detalle en la } \\
\text { figura } 1\end{array}$ & 5 & 5 & 5 & 5 & 3 & 4 & 4 & 4,43 \\
\hline $\begin{array}{l}\text { Actividad } 2 \text { - Ver detalle en la } \\
\text { figura } 1\end{array}$ & 5 & 5 & 5 & 5 & 3 & 4 & 4 & 4,43 \\
\hline $\begin{array}{l}\text { Actividad } 3 \text { - Ver detalle en la } \\
\text { figura } 1\end{array}$ & 5 & 4 & 5 & 5 & 3 & 4 & 4 & 4,29 \\
\hline $\begin{array}{l}\text { Actividad } 4 \text { - Ver detalle en la } \\
\text { figura } 1\end{array}$ & 5 & 4 & 5 & 5 & 3 & 4 & 4 & 4,29 \\
\hline $\begin{array}{l}\text { Promedio de la buena práctica y su } \\
\text { nivel de impacto en las } 4 \\
\text { actividades }\end{array}$ & 5 & 4,5 & 5 & 5 & 3 & 4 & 4 & 4,36 \\
\hline
\end{tabular}

práctica 2 obtiene un promedio de 4,50 y las prácticas 6 y 7 logran un promedio de 4,00 , lo que indica que el grado de influencia de estas 3 prácticas, con respecto a las actividades virtuales, es aceptable. Cuarto, la práctica 5 (enfatizar el tiempo de dedicación a la tarea) alcanza un promedio de 3,00, lo que implica que esta práctica logra un impacto medio en las 4 actividades de educación virtual. Esta práctica no logra convencer al docente de su adopción dentro del proceso de planificación y diseño curricular del curso. Finalmente, el indicador clave representado por $\mathrm{P}_{n+1}, m+1$ proveniente de la tabla 2 es de 4,36, lo que significa que en términos generales las

Tabla 5

Promedio de promedios de los estudiantes

\begin{tabular}{|c|c|c|c|c|c|}
\hline \multirow[t]{2}{*}{ Casos } & \multicolumn{4}{|c|}{ Actividades de educación virtual } & \multirow{2}{*}{$\begin{array}{l}\text { Promedio por caso o estudiante } \\
\text { participante }\end{array}$} \\
\hline & Actividad 1 & Actividad 2 & Actividad 3 & Actividad 4 & \\
\hline Estudiante 1 & 5 & 4 & 4 & 5 & 4,5 \\
\hline Estudiante 2 & 4 & 5 & 5 & 3 & 4,25 \\
\hline Estudiante 3 & 5 & 5 & 5 & 5 & 5 \\
\hline Estudiante 4 & 5 & 5 & 5 & 5 & 5 \\
\hline Estudiante 5 & 5 & 4 & 4 & 5 & 4,5 \\
\hline Estudiante 6 & 5 & 4 & 5 & 5 & 4,75 \\
\hline Estudiante 7 & 5 & 5 & 5 & 5 & 5 \\
\hline Estudiante 8 & 4 & 3 & 4 & 4 & 3,75 \\
\hline Estudiante 9 & 5 & 5 & 4 & 5 & 4,75 \\
\hline Estudiante 10 & 3 & 4 & 3 & 3 & 3,25 \\
\hline Promedio por actividad & 4,60 & 4,40 & 4,40 & 4,50 & 4,48 \\
\hline
\end{tabular}




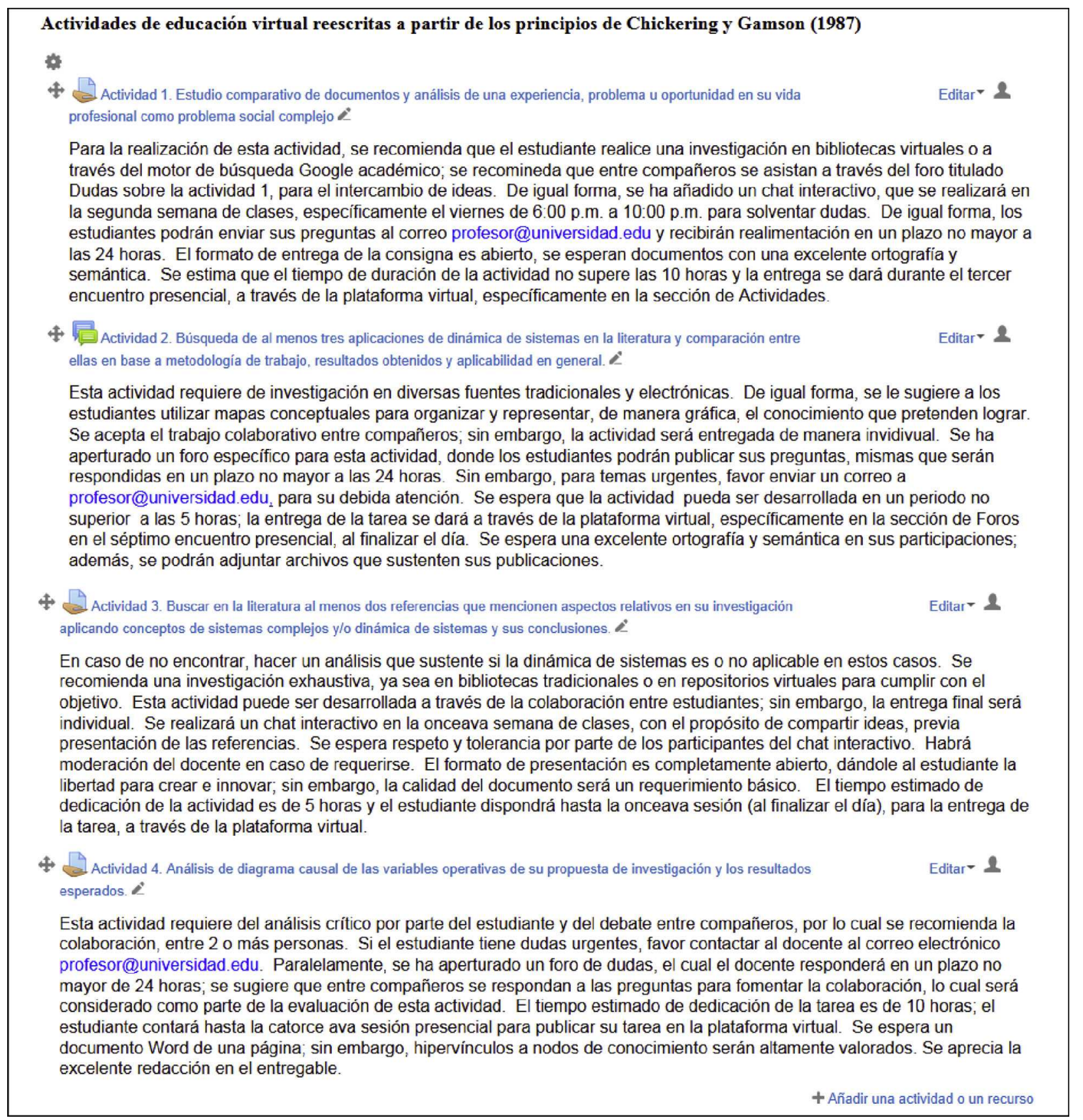

Figura 3. Actividades de aprendizaje en modalidad virtual

actividades de aprendizaje se redactaron de tal manera que cumplen con las 7 prácticas educativas de Chickering y Gamson (1987). La tabla 5 presenta el promedio de promedios de los estudiantes.

A partir de la lectura de la tabla 5 se obtienen los siguientes resultados: primero, los estudiantes 3, 4 y 7 logran el mejor impacto en sus aprendizajes a través de las actividades de educación virtual, ya que sus respectivos promedios individuales equivalen a 5,00. Segundo, el estudiante 10 es quien, en su opinión, logra el menor impacto en sus aprendizajes ya que el promedio tras evaluar las 4 actividades de educación virtual es solo de 3,25. Tercero, el estudiante 8 evalúa desde su perspectiva con un promedio de 3,75 sus aprendizajes a través del desarrollo de las actividades de educación virtual. Cuarto, las actividades 2 y 3 obtuvieron un promedio de 4,40 , la actividad 4 de 4,50 y la actividad 1 de 4,60 , lo que significa que las 4 actividades, en términos generales, recibieron una buena calificación de los estudiantes participantes y finalmente, el indicador clave representado por promedio de promedios proveniente de la tabla 3 es de 4,48, lo que significa que las 4 actividades de educación virtual impactaron en buena medida los aprendizajes de los estudiantes.

\section{Discusión y conclusiones}

Por un lado, se ha explorado descriptivamente el potencial de la educación virtual en la educación superior como alternativa de calidad en el proceso de enseñanza y aprendizaje, a través del presente estudio de caso. El docente investigador logró configurar las actividades de aprendizaje en la plataforma virtual de la universidad suministrando los parámetros necesarios para que el estudiante pudiera leer, comprender, desarrollar y entregar sus tareas virtuales, de acuerdo con lo estipulado en el cronograma de entregables. 
Por otro lado, el empleo del modelo de buenas prácticas educativas de Chickering y Gamson (1987) en la redacción de las actividades que se desarrollan en modalidad virtual fue factible, ya que el docente logró transformar 4 actividades genéricas virtuales en 4 actividades virtuales que incorporan los 7 principios del citado modelo. Además, la redacción de estas actividades según el estilo de buenas prácticas y el impacto de estas actividades en los aprendizajes fueron autoevaluadas por el docente y evaluadas por los alumnos durante la investigación.

De igual forma, se determinaron los impactos de las buenas prácticas en la didáctica y el aprendizaje, a partir del cálculo de $\mathrm{P}_{n+1, m+1}$ (tabla 2) y del promedio de promedios (tabla 3). Para el primer indicador, se lograron los datos requeridos por parte del docente para aplicar la fórmula del instrumento $1 \mathrm{y}$ para el segundo indicador, se lograron los datos requeridos por parte de los estudiantes para aplicar la fórmula del instrumento 3.

En cuanto a las conclusiones del presente estudio, se puede señalar que la incorporación de buenas prácticas educativas en la planificación, diseño e implementación curricular, específicamente en las actividades virtuales, le imprime dinamismo a la didáctica del docente. Las buenas prácticas de Chickering y Gamson (1987) refuerzan las actividades de aprendizaje ya que incorporan en sus narrativas aspectos que el docente podría obviar al momento de realizar su planificación y diseño curricular.

Además, la educación virtual en la educación superior es un medio efectivo para poner en marcha buenas prácticas educativas, ya que a través de las plataformas virtuales se pueden publicar las actividades con narrativas bien definidas y estas a la vez se pueden actualizar conforme a las necesidades del curso. En la figura 3 se muestra el botón que permite la edición de la actividad en la plataforma virtual (Jarauta, 2012; López, 2014). Cabe destacar, que los resultados de la investigación servirán de fundamento a las universidades para sustentar sus carreras virtuales y así aumentar su oferta académica. Para el caso específico de Panamá, las universidades particulares podrán incorporar estos indicadores claves como parte de la justificación de su oferta virtual al momento de someter estas carreras a consideración de las autoridades oficiales panameñas.

Existen varias líneas de investigación que se recomiendan para su estudio en el campo de la Ingeniería para la educación virtual en la educación superior, entre las que destacan: la validación de los instrumentos y de la metodología empleada en el presente manuscrito por expertos en Educación, la capacitación a docentes con un enfoque en competencias, los aprendizajes personalizados, la investigación científica, la formulación, evaluación y administración de proyectos, entre otras.

Durante el periodo en que se realizó el estudio, el profesor investigador tenía asignados 2 cursos de Maestría. El primer curso se ofrecería durante un periodo semestral y el segundo en un periodo trimestral. Por ende, el equipo investigador sugirió que se trabajara con el curso semestral para contar con más tiempo para el estudio. Finalmente, se reconoce que el tamaño de la muestra $(n=10)$ es limitado y no se pretende la generalización de las conclusiones. Por ende, es necesario ampliar la muestra y recoger más datos a partir de la replicación del método propuesto en futuras investigaciones.

\section{Conflicto de intereses}

Los autores declaran no tener ningún conflicto de intereses.

\section{Referencias bibliográficas}

Asociaciones de Educación a Distancia (AED). (2014). Asociaciones de educación a distancia: Mundiales, regionales y nacionales, sistematización, catalogación y descripción de recursos electrónicos enmarcados en dicha tipología. Centro Universitario de Educación a Distancia de la UNESCO [consultado 15
Nov 2014]. Disponible en http://www.uned.es/catedraunesco-ead/silviaalgarte/ nacionales.htm

Asociación Nacional de Universidades e Instituciones de Educación Superior (ANUIES)-UNESCO. (2004). La educación superior virtual en América Latina y el Caribe. México: Serie Memorias. Colección Biblioteca de la Educación Superior. ANUIES, UNESCO, IESALC.

Alexander, R. (1997). Policy and practice in primary education: Local initiative, national agenda. London: Routledge.

Babb, S., Stewart, C., y Johnson, R. (2013). Applying the seven principles for good practice in undergraduate education to blended learning environments. Practical applications and experiences in $\mathrm{K}-20$ blended learning environments, pp.192.

Bangert, A. W. (2004). The seven principles of good practice: A framework for evaluating on-line teaching. The Internet and Higher Education, 7(3), 217-232.

Cabero, J. y Romero, R. (2010). Análisis de buenas prácticas del e-learning en las universidades andaluzas. Educación y Cultura en la Sociedad de la Información. Revista Electrónica Teoría de la Educación, 11(1), 283-309.

Cakiroglu, U. (2014). Evaluating students' perspectives about virtual classrooms with regard to Seven principles of good practice. South African Journal of Education, 34(2), 1-19.

Chickering, A. W. y Gamson, Z. F. (1987). Seven principles for good practice in undergraduate education. AAHE Bulletin, 39(1), 3-7.

Coffield, F. y Edward, S. H. (2009). Rolling out good, best and excellent practice What next? Perfect practice? British Educational Research Journal, 35(3), 371-390.

Comisión Técnica de Fiscalización de Panamá (CTF). (2014). Lista Oficial de Planes y Programas de Estudios Aprobados (Orden Cronológico por Universidad) al 25 de febrero del 2014. Comisión Técnica de Fiscalización de Panamá [consultado 20 Jul 2014]. Disponible en http://www.ctf.ac.pa/planes_univ_aprobados.htm

Consejo Nacional de Evaluación y Acreditación Universitaria de Panamá (CONEAUPA). (2014). Normativa, leyes, decretos, y resoluciones. CONEAUPA [consultado 20 Jul 2014]. Disponible en http://coneaupa.wix.com/coneaupa/ normativa

De Pablos, J. y Jiménez, R. (2007). Buenas prácticas con TIC apoyadas en las políticas educativas: Claves conceptuales y derivaciones para la formación en competencias ECTS. Revista Latinoamericana de Tecnología Educativa, 6(2), 15-28.

Dixon, M. D. (2012). Creating effective student engagement in online courses: What do students find engaging? Journal of the Scholarship of Teaching and Learning, 10(2), 1-13.

Durall, E., Gros, B., Maina, M. F., Johnson, L. y Adams, S. (2012). Perspectivas tecnológicas: Educación superior en Iberoamérica 2012-2017. The New Media Consortium y la Universitat Oberta de Catalunya [consultado 10 Nov 2014]. Disponible en http://openaccess.uoc.edu/webapps/o2/bitstream/10609/17021/6/horizon_ iberoamerica_2012_ESP.pdf

Europa (2014). EUR-Lex. El acceso al Derecho de la Unión Europea (UE). Publicado por el Diario Oficial de la UE [consultado 20 Ago 2014]. Disponible en http://eur-lex.europa.eu/homepage.html

Echeverría, B. (2002). Gestión de la competencia de acción profesional. Barcelona: Universidad de Barcelona.

Fernández, K. y Vallejo, A. (2014). La educación en línea: Una perspectiva basada en la experiencia de los países. Revista de Educación y Desarrollo, 29, 29-39.

García, L. (1999). Historia de la educación a distancia. Revista Iberoamericana de Educación a Distancia (RIED), 2(1), 11-40.

Graham, C., Cagiltay, K., Lim, B., Craner, J. y Duffy, T. M. (2001). Seven principles of effective teaching: A practical lens for evaluating online courses. The Technology Source, 30(5), 50.

Grupo de Investigación y Multimedia. Universidad Autónoma de Barcelona. (2014). Red de Buenas Prácticas 2.0. Revista DIM [consultado 25 Nov 2014]. Disponible en https://dl.dropboxusercontent.com/u/20875810/dim/revistaDIM29/ revistanew.htm

Hammer, M. (1990). Reengineering work: Don't automate, obliterate. Boston: Harvard. Hutchins, H. M. (2003). Instructional immediacy and the seven principles: Strategies for facilitating online courses. Online Journal of Distance Learning Administration, 6(3), $1-13$.

Jarauta, B. (editor).(2012). Pensando en el futuro de la educación: Una nueva escuela para el siglo XXII (Vol. 39). Barcelona: Graó.

Junta de Andalucía. Consejería de Educación. (2012). Guía sobre buenas prácticas docentes para el desarrollo en el aula de las competencias básicas del alumnado. Junta de Andalucía [consultado 5 Ago 2015]. Disponible en http://www. juntadeandalucia.es/averroes/ceip_albinas/documentos/Guia_buenas_practicas. docentes.pdf

López, R. M. (2014). Sloodle. Conexión de entornos de aprendizaje. Barcelona: Editorial UOC.

Luque, M. (2004). Visión americana de la perspectiva social del e-learning en la educación superior. Revista de Universidad y Sociedad del Conocimiento (RUSC), $128-142$.

Pastor, C. A. y Santaolalla, R. C. (2005). Viabilidad de las propuestas metodológicas para la aplicación del crédito europeo por parte del profesorado de las universidades españolas, vinculadas a la utilización de las TIC en la docencia y la investigación. Revista de Educación, 337, 71-97.

Programa de Promoción de la Reforma Educativa en América Latina y el Caribe (PREAL) (2014). Buenas prácticas del PREAL. Programa de Buenas Prácticas [consultado 4 Ago 2014]. Disponible en http://www.preal.org/Practica.asp?Id_ Practica $=8$

Rama, C. (2006). La tercera reforma de la educación superior en América Latina. Buenos Aires: Fondo de Cultura Económica.

Rama, C. A. (2014). University virtualisation in Latin America. RUSC. Universities and Knowledge Society Journal, 11(3), 32-41. 
Red Telescopi (2014). Red de Observatorios de Buenas Prácticas de Dirección Estratégica Universitaria en Latinoamérica y Europa. Programa ALFA III [consultado 20 Ago 2014]. Disponible en http://telescopi.upc.edu/

Silvio, J. (2000). La virtualización de la Universidad: ¿Cómo transformar la educación superior con la tecnología? Caracas: IESALC/UNESCO.

Silvio, J. (2006). Hacia una educación virtual de calidad, pero con equidad y pertinencia. Revista de Universidad y Sociedad del Conocimiento, 3(1), $1-14$

Suárez, J. M. y Anaya, D. (2004). Educación a distancia y presencial: Diferencias en los componentes cognitivo y motivacional de estudiantes universitarios. RIED. Revista Iberoamericana de Educación a Distancia, 7(1-2), $65-75$
The Global University Network for Innovation. (2014). Good practices. The Global University Network for Innovation [consultado 20 Ago 2014]. Disponible en http://www.guninetwork.org/guni.heiobs/good-practices

Tobin, T. J. (2004). Best practices for administrative evaluation of online faculty. Online Journal of Distance Learning Administration, 7(2). Disponible en http://www.westga.edu/ distance/ojdla/summer72/tobin72.html.

Tunnermann, C. (2008). La educación superior en América Latina y el Caribe. Diez años después de la Conferencia Mundial de 1998. UNESCO. IESALC.

Van Haecht, A. (1998). Les politiques éducatives, figure exemplaire des politiques publiques? Education et societés, 1, 21-46.

Zabalza, M. (2012). El estudio de las buenas prácticas docentes en la enseñanza universitaria. Revista de Docencia Universitaria, 10(1), 17-42. 